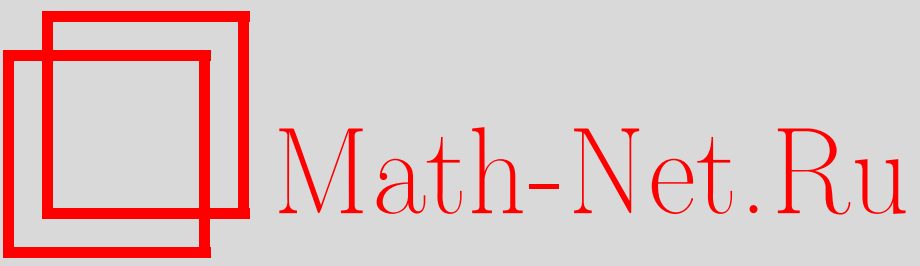

К. Верховен, М. Музетте, Р. Конт, Общее решение для гамильтонианов с добавленными кубичными и четвертичными потенциалами, ТМФ, 2003, том 134 , номер 1, 148-159

DOI: https://doi.org/10.4213/tmf147

Использование Общероссийского математического портала Math-Net.Ru подразумевает, что вы прочитали и согласны с пользовательским соглашением

http://www.mathnet.ru/rus/agreement

Параметры загрузки:

IP : 44.207 .124 .84

26 апреля 2023 г., 16:47:11 


\section{ОБШЕЕ РЕШЕНИЕ ДЛЯ ГАМИЛЬТОНИАНОВ С ДОБАВЛЕННЫМИ КУБИЧНЫМИ И ЧЕТВЕРТИЧНЫМИ ПОТЕНЦИАЛАМИ}

Двухчастичный гамильтониан с добавленными потенциалом четвертого порядка и дополнительными линейными и неполиномиальными членами проинтегрирован с использованием гиперэллиптических функций в случаях $1: 6: 1$ и $1: 6: 8$, интегрируемых по Лиувиллю.

Ключевые слова: гамильтониан Хенона-Хейлеса, гамильтоновы системы, разделение переменных, гиперэллиптические функции, солитонные уравнения.

\section{1. ВВЕДЕНИЕ}

Как известно, обобщенный гамильтониан Хенона-Хейлеса

$$
H=\frac{1}{2}\left(P_{X}^{2}+P_{Y}^{2}+c_{1} X^{2}+c_{2} Y^{2}\right)+a X Y^{2}-\frac{b}{3} X^{3}+\mu Y^{-2}
$$

для любого $\mu$ оказывается интегрируемым по Лиувиллю [1]-[3] для трех наборов значений $\left(b / a, c_{1}, c_{2}\right)$, которые связаны [4] с соответствуюшими стационарными редукциями

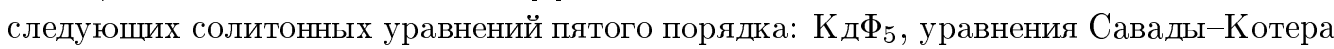
(CK) и уравнения Каупа-Купершмидта (KK). Каноническое преобразование [5] между случаями СK и КK при $\mu \neq 0$ позволяет [6] разделить переменные в уравнениях Гамильтона-Якоби и получить общее решение уравнений движения в терминах гиперэллиптических функций.

Известно, что гамильтониан системы двух частиц с потенциалом четвертого порядка,

$$
H=\frac{1}{2}\left(P_{X}^{2}+P_{Y}^{2}\right)-\frac{1}{2}\left(a X^{2}+b Y^{2}\right)+C X^{4}+B X^{2} Y^{2}+A Y^{4},
$$

оказывается интегрируемым по Лиувиллю в следующих четырех случаях [7]-[9]: 1) $A$ : $B: C=1: 2: 1, a, b$ произвольны; 2) $A: B: C=1: 6: 1, a=b ; 3) A: B: C=1:$

*Dienst Theoretische Natuurkunde, Vrije Universiteit Brussel, Brussels, Belgium. E-mail: cverhoev@vub.ac.be, mmusette@vub.ac.be

${ }^{\dagger}$ Service de Physique de Létat Condensé, CEA, Saclay, France.

E-mail: Conte@drecam.saclay.cea.fr 
$6: 8, a=4 b$; 4) $A: B: C=1: 12: 16, a=4 b$. Были также получены расширения гамильтониана (2), включаюшие некоторые линейные и неполиномиальные члены и при этом сохраняюшие свойство интегрируемости по Лиувиллю [9], [10], что подтверждает их связь с некоторыми интегрируемыми солитонными уравнениями [11], [12].

Случай 1 с дополнительными обратными членами второго порядка эквивалентен редукции типа движущейся волны [13] системы Манакова двух связанных уравнений НШ. Это отвечает частному случаю двух частиц из расширенной системы Гарнье [14], которая была проинтегрирована Войцеховским [15]. Случай 4 связан [5] с присоединенной системой Кд

Авторы работ [5], [17] показали, что случаи 2 и 3 с некоторыми добавленными линейными и обратными квадратичными членами можно связать с системой Хироты-Сатсумы [18]

$$
r_{t}=\frac{1}{2} r_{x x x}+3 r r_{x}-6 s s_{x}, \quad s_{t}=-s_{x x x}-3 r s_{x}
$$

а также с другой связанной системой типа $\mathrm{K} д \Phi$,

$$
\begin{aligned}
f_{t}= & -\frac{1}{2}\left(2 f_{x x x}+3 f f_{x x}+3 f_{x}^{2}-3 f^{2} f_{x}+6 f g_{x}+6 g f_{x}\right) \\
g_{t}= & \frac{1}{4}\left(2 g_{x x x}+12 g g_{x}+6 f g_{x x}+12 g f_{x x}+18 f_{x} g_{x}-6 f^{2} g_{x}+\right. \\
& \left.+3 f_{x x x x}+3 f f_{x x x}+18 f_{x} f_{x x}-6 f^{2} f_{x x}-6 f f_{x}^{2}\right) .
\end{aligned}
$$

В данной работе каноническое преобразование [5], связываюшее две расширенные системы, используется для того чтобы применить к этим системам метод, использованный ранее в случаях систем CK и KK уравнения (1) для определения и разделения переменных в уравнении Гамильтона-Якоби. Уравнения движения для случаев 2 и 3 гамильтониана (2) с добавочными линейными и обратными квадратичными членами при этом интегрируются в терминах гиперэллиптических функций.

В разделе 2 рассматриваются интегрируемые случаи гамильтониана Хенона-Хейлеса (1), связанные с уравнениями СК и КК при $\mu \neq 0$, что объясняет метод, примененный нами в работе [6] для интегрирования соответствующих уравнений движения. В разделе 3 рассматривается гамильтониан (2) с дополнительным потенциалом четвертого порядка для случаев 2 и 3 и описывается ранее построенное каноническое преобразование [5], которое связывает случай $1: 6: 1$ (с дополнительными членами вида $\alpha X^{-2}+$ $\beta Y^{-2}$ ) и случай $1: 6: 8$ (с дополнительными членами вида $\left.\gamma X+\delta Y^{-2}\right)$.

Результаты разделов 4 и 5 , по нашему мнению, являются новыми. В разделе 4 явно интегрируются уравнения движения в случае $1: 6: 8$ при $\delta \neq 0, \gamma=0$. В разделе 5 проинтегрирован случай $1: 6: 1$ с параметрами $\alpha=\beta \neq 0$ и использовано каноническое преобразование, чтобы привести эти решения к решениям случая $1: 6: 8$ при $\delta=0$, $\gamma \neq 0$. 


\section{2. ИНТЕГРИРОВАНИЕ КУБИЧНОГО ГАМИЛЬТОНИАНА ХЕНОНА-ХЕЙЛЕСА}

Рассмотрим гамильтонианы Хенона-Хейлеса для уравнений СК и KK, имеюшие соответствуюшие вторые интегралы $K_{2}^{2}$ и $k_{2}^{2}$ и уравнения движения [9], [10]:

$$
\begin{array}{ll}
\mathrm{CK}: \quad & \frac{b}{a}=-1, \quad c_{1}=c_{2}, \quad a=\frac{1}{2}, \quad U=X+c_{2}, \quad V=Y, \quad c=c_{1} c_{2}, \\
& H \equiv K_{1}=\frac{1}{2}\left(P_{U}^{2}+P_{V}^{2}\right)+\frac{1}{2} U V^{2}+\frac{1}{6} U^{3}-\frac{c}{2} U+\frac{\mu}{8 V^{2}}, \\
& K_{2}^{2}=K_{2,0}^{2}+\frac{2}{3} \mu U+\mu \frac{P_{U}^{2}}{V^{2}}, \\
& K_{2,0}=-2 P_{U} P_{V}-U^{2} V-\frac{V^{3}}{3}+c V, \\
& U^{\prime \prime}=-\frac{1}{2}\left(V^{2}+U^{2}\right)-\frac{c}{2}, \quad V^{\prime \prime}=-U V+\frac{\mu}{4 V^{3}}, \\
\mathrm{KK}: \quad \frac{b}{a}=-16, \quad c_{1}=16 c_{2}, \quad a=\frac{1}{4}, \quad c=c_{1} c_{2}, \quad u=X+2 c_{2}, \quad V=Y, \\
H & \equiv k_{1}=\frac{1}{2}\left(p_{u}^{2}+p_{v}^{2}\right)+\frac{1}{4} u v^{2}+\frac{4}{3} u^{3}-c u+\frac{1}{2} \frac{\mu}{v^{2}}, \\
k_{2}^{2} & =k_{2,0}^{2}+\frac{\mu}{3} u+2 \mu \frac{p_{v}^{2}}{v^{2}}+\frac{\mu^{2}}{v^{4}}, \\
k_{2,0}^{2} & =p_{v}^{4}-\frac{1}{72} v^{6}-\frac{1}{12} u^{2} v^{4}+u p_{v}^{2} v^{2}-\frac{1}{3} p_{u} p_{v} v^{3}+\frac{c}{12} v^{4}, \\
u^{\prime \prime} & =-\frac{1}{4} v^{2}-4 u^{2}+c, \quad v^{\prime \prime}=-\frac{1}{2} u v+\frac{\mu}{v^{3}} .
\end{array}
$$

Эти уравнения эквивалентны стационарной редукции $\xi=x-c t$ следуюших двух интегрируемых УрЧП:

$$
\begin{aligned}
& \text { CK: } U_{t}+\left(U_{x x x x}+5 U U_{x x}+\frac{5}{3} U^{3}\right)_{x}=0, \\
& \text { KK: } \quad u_{t}+\left(u_{x x x x}+10 u u_{x x}+\frac{20}{3} u^{3}+30 u_{x}^{2}\right)_{x}=0 .
\end{aligned}
$$

Оба уравнения связаны с другим интегрируемым УрЧП пятого порядка [19],

$$
w_{t}+\left(w_{4 x}-5 w_{x} w_{x x}-5 w^{2} w_{x x}-5 w w_{x}^{2}+w^{5}\right)_{x}=0,
$$

с помощью преобразования Миуры

$$
U=w_{x}-w^{2}, \quad u=-w_{x}-\frac{1}{2} w^{2} .
$$

Вычисляя стационарную редукцию уравнения (17) для $w$ как функции от $V, V^{\prime}$ и $v, v^{\prime}$ и определяя выражения

$$
\begin{aligned}
\lambda^{2}= & -\mu, \quad \Gamma=6\left(V K_{2,0}+\lambda P_{U}\right) \\
\Omega= & 48\left(3 v^{4} k_{2,0}^{2}+6 \lambda u v^{5} p_{v}+12 \lambda p_{v}^{3} v^{3}-\lambda v^{6} p_{u}+\right. \\
& \left.+3 \lambda^{2} u v^{4}+18 \lambda^{2} v^{2} p_{v}^{2}+12 \lambda^{3} v p_{v}+3 \lambda^{4}\right),
\end{aligned}
$$


можно получить следуюшее каноническое преобразование [5], [20]:

$$
\begin{aligned}
u= & -\frac{3}{2}\left(-\frac{P_{V}}{V}+\frac{\lambda}{2 V^{2}}\right)^{2}-U, \quad v=\frac{\sqrt{\Gamma}}{V}, \\
p_{u}= & \frac{1}{V^{3}}\left(3 P_{V}^{3}+3 U V^{2} P_{V}-P_{U} V^{3}\right)- \\
& -\frac{3 \lambda}{2 V^{6}}\left(U V^{4}+3 V^{2} P_{V}^{2}-\frac{3}{2} \lambda V P_{V}+\frac{\lambda^{2}}{4}\right), \\
p_{v}= & \frac{1}{4 V^{2}}\left(-2 P_{V}+\frac{\lambda}{V}\right) \sqrt{\Gamma}-\lambda \frac{V}{\sqrt{\Gamma}}, \\
U= & -6\left(\frac{p_{v}}{v}+\frac{\lambda}{v^{2}}\right)^{2}-u, \quad V=\frac{\sqrt{\Omega}}{2 v^{4}}, \\
P_{U}= & \frac{1}{v^{3}}\left(12 p_{v}^{3}+6 u v^{2} p_{v}-v^{3} p_{u}\right)+\frac{3 \lambda}{v^{6}}\left(2 u v^{4}+12 v^{2} p_{v}^{2}+12 \lambda v p_{v}+4 \lambda^{2}\right), \\
P_{V}= & -\frac{1}{v^{5}}\left(p_{v}+\frac{\lambda}{v}\right) \sqrt{\Omega}+\lambda \frac{v^{4}}{\sqrt{\Omega}} .
\end{aligned}
$$

Для случая $\mu=0$ можно представить выражения (22)-(24) для $U, V, P_{U}, P_{V}$ в терминах переменных, которые задают разделение переменных в гамильтониане СK,

$$
\begin{array}{ll}
Q_{1}=U+V, & P_{1}=\frac{1}{2}\left(P_{U}+P_{V}\right) \\
Q_{2}=U-V, & P_{2}=\frac{1}{2}\left(P_{U}-P_{V}\right) .
\end{array}
$$

Это задает следующую замену переменных [21]:

$$
\begin{array}{ll}
q_{1}=-6 \frac{p_{v}^{2}-k_{2,0}}{v^{2}}-u, & p_{1}=\frac{1}{2 v^{3}}\left(12 p_{v}^{3}+6 u v^{2} p_{v}-v^{3} p_{u}-12 p_{v} k_{2,0}\right), \\
q_{2}=-6 \frac{p_{v}^{2}+k_{2,0}}{v^{2}}-u, & p_{2}=\frac{1}{2 v^{3}}\left(12 p_{v}^{3}+6 u v^{2} p_{v}-v^{3} p_{u}+12 p_{v} k_{2,0}\right),
\end{array}
$$

которую можно применить к гамильтониану KK (11) с учетом того, что $k_{2,0}$ не является константой движения при $\mu \neq 0$. Тогда получим

$$
\begin{aligned}
H & \equiv k_{1}=p_{1}^{2}+p_{2}^{2}+\frac{1}{12}\left(q_{1}^{3}+q_{2}^{3}\right)-\frac{c}{4}\left(q_{1}+q_{2}\right)+\frac{\mu}{24} \frac{q_{1}-q_{2}}{k_{2,0}}, \\
k_{2,0} & =2\left(p_{2}^{2}-p_{1}^{2}\right)+\frac{1}{6}\left(q_{2}^{3}-q_{1}^{3}\right)-\frac{c}{2}\left(q_{2}-q_{1}\right), \\
q_{1}^{\prime} & =2 p_{1}+\frac{\mu}{6} \frac{\left(q_{1}-q_{2}\right) p_{1}}{k_{2,0}^{2}}, \\
q_{2}^{\prime} & =2 p_{2}-\frac{\mu}{6} \frac{\left(q_{1}-q_{2}\right) p_{2}}{k_{2,0}^{2}} .
\end{aligned}
$$


Пользуясь этими новыми координатами и вводя выражения

$$
f\left(q_{i}, p_{i}\right)=2 p_{i}^{2}+\frac{1}{6} q_{i}^{3}-\frac{c}{2} q_{i}, \quad i=1,2,
$$

в формулах (27) и (28), получаем, что уравнение Гамильтона-Якоби допускает разделение переменных при произвольном $\mu$.

В терминах переменных (26) второй инвариант $k_{2}^{2}$ может быть записан в двух эквивалентных формах:

$$
\begin{aligned}
& k_{2}^{2}=-\frac{\mu}{3} q_{1}+\left(k_{2,0}+\frac{\mu}{12} \frac{q_{1}-q_{2}}{k_{2,0}}\right)^{2}, \\
& k_{2}^{2}=-\frac{\mu}{3} q_{2}+\left(k_{2,0}-\frac{\mu}{12} \frac{q_{1}-q_{2}}{k_{2,0}}\right)^{2},
\end{aligned}
$$

которые после исключения $\mu\left(q_{1}-q_{2}\right) / k_{2,0}$ и $k_{2,0}$ с использованием формул $(27)$ и (28) принимают вид

$$
\begin{aligned}
& k_{2}^{2}=-\frac{\mu}{3} q_{1}+\left(-4 p_{1}^{2}-\frac{q_{1}^{3}}{3}+c q_{1}+2 k_{1}\right)^{2}, \\
& k_{2}^{2}=-\frac{\mu}{3} q_{2}+\left(4 p_{2}^{2}+\frac{q_{2}^{3}}{3}-c q_{2}-2 k_{1}\right)^{2} .
\end{aligned}
$$

Исключая далее $p_{1}$ из уравнений $(34)$ и $(29)$ и $p_{2}$ из уравнений $(35)$ и $(30)$, получаем

$$
\begin{aligned}
& q_{1}^{\prime}=\sqrt{2 k_{1}-\frac{q_{1}^{3}}{3}+c q_{1}-\sqrt{k_{2}^{2}+\frac{\mu}{3} q_{1}}}\left(1+\frac{\mu}{3} \frac{q_{1}-q_{2}}{\left(\sqrt{k_{2}^{2}+\frac{\mu}{3} q_{2}}+\sqrt{k_{2}^{2}+\frac{\mu}{3} q_{1}}\right)^{2}}\right) \\
& q_{2}^{\prime}=\sqrt{2 k_{1}-\frac{q_{2}^{3}}{3}+c q_{2}+\sqrt{k_{2}^{2}+\frac{\mu}{3} q_{2}}}\left(1-\frac{\mu}{3} \frac{q_{1}-q_{2}}{\left(\sqrt{k_{2}^{2}+\frac{\mu}{3} q_{2}}+\sqrt{k_{2}^{2}+\frac{\mu}{3} q_{1}}\right)^{2}}\right)
\end{aligned}
$$

При $\mu \neq 0$, полагая

$$
s_{1}=\sqrt{3 \frac{k_{2}^{2}}{\mu}+q_{1}}, \quad s_{2}=-\sqrt{3 \frac{k_{2}^{2}}{\mu}+q_{2}}
$$

и определяя выражение

$$
P(s)=2 k_{1}-\frac{1}{3}\left(s^{2}-3 \frac{k_{2}^{2}}{\mu}\right)^{3}+c\left(s^{2}-3 \frac{k_{2}^{2}}{\mu}\right)-\sqrt{\frac{\mu}{3}} s,
$$

можно привести уравнения (36), (37) к виду

$$
s_{1}^{\prime}=\frac{\sqrt{P\left(s_{1}\right)}}{s_{1}-s_{2}}, \quad s_{2}^{\prime}=-\frac{\sqrt{P\left(s_{2}\right)}}{s_{1}-s_{2}} .
$$


Эти уравнения можно решить путем обращения гиперэллиптических интегралов

$$
\begin{aligned}
& \int_{\infty}^{s_{1}} \frac{d s}{\sqrt{P(s)}}+\int_{\infty}^{s_{2}} \frac{d s}{\sqrt{P(s)}}=k_{3} \\
& \int_{\infty}^{s_{1}} \frac{s d s}{\sqrt{P(s)}}+\int_{\infty}^{s_{2}} \frac{s d s}{\sqrt{P(s)}}=\xi+k_{4}
\end{aligned}
$$

что и дает обшее решение системы KK:

$$
u=-\frac{1}{2}\left(s_{1}^{2}+s_{2}^{2}\right)+\frac{3}{\mu} k_{2}^{2}-\frac{3}{2}\left(\frac{s_{1}^{\prime}+s_{2}^{\prime}}{s_{1}+s_{2}}\right)^{2}, \quad v^{2}=\frac{2 \sqrt{3 \mu}}{s_{1}+s_{2}} .
$$

При $\mu=0$ можно легко получить из $(36),(37)$ и (26) известное решение [21] с эллиптическими функциями Вейерштрасса:

$$
u=6\left(\wp_{1}+\wp_{2}\right)-\frac{3}{2}\left(\frac{\wp_{1}^{\prime}-\wp_{2}^{\prime}}{\wp_{1}-\wp_{2}}\right)^{2}, \quad v^{2}=\frac{k_{2,0}}{\wp_{2}-\wp_{1}},
$$

где функции юi удовлетворяют уравнениям

$$
\begin{gathered}
\wp_{i}^{\prime 2}=4 \wp_{i}^{3}-g_{2} \wp_{i}-g_{3}^{(i)}, \quad i=1,2, \\
g_{2}=\frac{c}{12}, \quad g_{3}^{(1)}=-\frac{1}{144}\left(2 k_{1,0}-k_{2,0}\right), \quad g_{3}^{(2)}=-\frac{1}{144}\left(2 k_{1,0}+k_{2,0}\right) .
\end{gathered}
$$

В случае CK общее решение, получающееся из переменных (43) и канонического преобразования (22), имеет вид

$$
\begin{aligned}
U & =\sqrt{-3}\left(s_{1}^{\prime}+s_{2}^{\prime}\right)+s_{1}^{2}+s_{1} s_{2}+s_{2}^{2}-\frac{3}{\mu} K_{2}^{2} \\
V^{2} & =-2 \sqrt{-3}\left(s_{1}+s_{2}\right)\left(s_{1} s_{1}^{\prime}+s_{2} s_{2}^{\prime}\right)+2\left(s_{1}+s_{2}\right)^{2}\left(s_{1}^{2}+s_{2}^{2}-\frac{9 K_{2}^{2}}{2 \mu}\right) .
\end{aligned}
$$

В частном случае $\mu=0$ это решение принимает простой вид

$$
U=-6\left(\wp_{1}+\wp_{2}\right), \quad V=-6\left(\wp_{1}-\wp_{2}\right) .
$$

\section{3. КАНОНИЧЕСКОЕ ПРЕОБРАЗОВАНИЕ МЕЖДУУ}

РАСШИРЕННЫМИ ГАМИЛЬТОНИАНАМИ $1: 6: 1$ И $1: 6: 8$

Рассмотрим теперь гамильтониан четвертого порядка (2) с дополнительными линейными и обратными квадратичными членами в следуюших двух случаях [9]:

$$
\begin{aligned}
1: 6: 1: \quad H \equiv K_{1}= & \frac{1}{2}\left(P_{U}^{2}+P_{V}^{2}\right)-\frac{1}{32}\left(U^{4}+6 U^{2} V^{2}+V^{4}\right)- \\
& -\frac{c}{2}\left(U^{2}+V^{2}\right)+\frac{1}{2}\left(\frac{\alpha}{U^{2}}+\frac{\beta}{V^{2}}\right)
\end{aligned}
$$




$$
\begin{aligned}
& K_{2}^{2}=K_{2,0}^{2}-\beta U^{2}-\alpha V^{2}+4\left(\alpha \frac{P_{V}^{2}}{U^{2}}+\beta \frac{P_{U}^{2}}{V^{2}}\right)+ \\
& +\frac{4 \alpha \beta}{U^{2} V^{2}}-4 c(\alpha+\beta) \\
& K_{2,0}=2 P_{U} P_{V}-\frac{1}{4} U V\left(U^{2}+V^{2}+8 c\right), \\
& U^{\prime}=P_{U}, \quad V^{\prime}=P_{V}, \\
& U^{\prime \prime}=\frac{1}{8} U^{3}+\frac{3}{8} U V^{2}+c U+\frac{\alpha}{U^{3}}, \\
& V^{\prime \prime}=\frac{1}{8} V^{3}+\frac{3}{8} U^{2} V+c V+\frac{\beta}{V^{3}} ; \\
& k_{2}^{2}=k_{2,0}^{2}+\frac{\delta^{2}}{v^{4}}-\gamma v^{2} u^{3}-\frac{\gamma}{2} u v^{4}-\frac{\delta}{2} u^{2}-2 \gamma^{2} v^{2}+2 \delta \frac{p_{v}^{2}}{v^{2}}- \\
& -\frac{\delta}{4} v^{2}+4 \gamma\left(v p_{u} p_{v}-u p_{v}^{2}\right)-4 \gamma \delta \frac{u}{v^{2}}-2 \delta c-4 c \gamma v^{2} u, \\
& k_{2,0}^{2}=\left(-p_{v}^{2}+\frac{1}{8} v^{4}+\frac{1}{4} u^{2} v^{2}-u v p_{v}+\frac{1}{2} p_{u} v^{2}+c v^{2}\right) \times \\
& \times\left(-p_{v}^{2}+\frac{1}{8} v^{4}+\frac{1}{4} u^{2} v^{2}+u v p_{v}-\frac{1}{2} p_{u} v^{2}+c v^{2}\right), \\
& u^{\prime}=p_{u}, \quad v^{\prime}=p_{v}, \\
& u^{\prime \prime}=2 u^{3}+\frac{3}{4} u v^{2}+4 c u+\gamma, \quad v^{\prime \prime}=\frac{1}{4} v^{3}+\frac{3}{4} u^{2} v+c v+\frac{\delta}{v^{3}},
\end{aligned}
$$

где $K_{1}, K_{2}$ и $k_{1}, k_{2}$ - интегралы движения двух соответствуюших систем.

Уравнения движения связаны со стационарной редукцией $\xi=x-c t$ соответствующих УрЧП (3) и (4), где

$$
\begin{aligned}
& r=-\frac{1}{4}\left(U^{2}+V^{2}+4 c\right), \quad s=\frac{1}{8}\left(U^{2}-V^{2}\right), \\
& f=u, \quad g=-\frac{1}{4}\left(v^{2}+2 u^{2}+2 p_{u}+4 c\right) .
\end{aligned}
$$

Как уравнение (3), так и уравнение (4) имеет пару Лакса четвертого порядка, и соответствуюшие операторы рассеяния $L$ и $\tilde{L}$ допускают факторизации [5], [17],

$$
\begin{aligned}
1: 6: 1: \quad L & \equiv\left(\partial_{x}^{2}+r+s\right)\left(\partial_{x}^{2}+r-s\right)= \\
& =\left(\partial_{x}-v_{1}\right)\left(\partial_{x}+v_{1}\right)\left(\partial_{x}+v_{2}\right)\left(\partial_{x}-v_{2}\right), \\
1: 6: 8: \quad \tilde{L} & \equiv\left(\partial_{x}^{2}+f \partial_{x}+f_{x}+g\right)\left(\partial_{x}-f \partial_{x}+g\right)= \\
& =\left(\partial_{x}+v_{1}\right)\left(\partial_{x}+v_{2}\right)\left(\partial_{x}-v_{2}\right)\left(\partial_{x}-v_{1}\right),
\end{aligned}
$$


и задают отображения Миуры,

$$
\begin{array}{ll}
1: 6: 1: & r=\frac{1}{2}\left(v_{1, x}-v_{2, x}-v_{1}^{2}-v_{2}^{2}\right), \quad s=\frac{1}{2}\left(v_{1, x}+v_{2, x}-v_{1}^{2}+v_{2}^{2}\right), \\
1: 6: 8: & f=v_{1}+v_{2}, \quad g=v_{1} v_{2}-v_{1, x},
\end{array}
$$

где $v_{1}$ и $v_{2}-$ решения системы УрЧП

$$
\begin{aligned}
& v_{1, t}=\frac{1}{8}\left(-2 v_{1, x x}-6 v_{2, x x}-12 v_{2} v_{2, x}-12 v_{1} v_{2, x}-12 v_{1} v_{2}^{2}+4 v_{1}^{3}\right)_{x}, \\
& v_{2, t}=\frac{1}{8}\left(-2 v_{2, x x}-6 v_{1, x x}+12 v_{1} v_{1, x}+12 v_{2} v_{1, x}-12 v_{2} v_{1}^{2}+4 v_{2}^{3}\right)_{x} .
\end{aligned}
$$

Из соотношений (62), (63), преобразований (58), (59) и уравнений движения (51), (52), (56), (57) следует, что условие стационарной редукции системы (64) можно разрешить относительно переменных $v_{1}$ и $v_{2}$ в терминах функций канонических переменных двух расширенных систем:

$$
\begin{aligned}
& 1: 6: 1: \quad v_{1}=-\frac{P_{U}}{U}+\frac{\sqrt{-\alpha}}{U^{2}}, \quad v_{2}=\frac{P_{V}}{V}+\frac{\sqrt{-\beta}}{V^{2}}, \\
& 1: 6: 8: \quad v_{1}=\frac{u}{2}+\frac{p_{v}}{v}+\frac{\sqrt{-\delta}}{v^{2}}, \quad v_{2}=\frac{u}{2}-\frac{p_{v}}{v}-\frac{\sqrt{-\delta}}{v^{2}} .
\end{aligned}
$$

Если ввести определения

$$
\begin{aligned}
& \alpha=-\kappa_{1}^{2}, \quad \beta=-\kappa_{2}^{2}, \quad \delta=-\left(\kappa_{2}-\kappa_{1}\right)^{2}, \quad \gamma=\frac{1}{2}\left(\kappa_{1}+\kappa_{2}\right), \\
& \Omega=-\frac{1}{4}\left(U^{2}+V^{2}\right)+\frac{2}{U V}\left(P_{U} P_{V}+\kappa_{2} \frac{P_{U}}{V}-\kappa_{1} \frac{P_{V}}{U}-\frac{\kappa_{1} \kappa_{2}}{U V}\right)-2 c, \\
& \Gamma_{\mp}=-4\left(\mp 2 p_{u}+\frac{1}{2} v^{2}+u^{2}-4 \frac{p_{v}^{2}}{v^{2}} \pm 4 \frac{u p_{v}}{v}+8\left(\kappa_{2}-\kappa_{1}\right) \frac{p_{v}}{v^{3}} \mp\right. \\
&\left.\mp 4\left(\kappa_{2}-\kappa_{1}\right) \frac{u}{v^{2}}-4 \frac{\left(\kappa_{2}-\kappa_{1}\right)^{2}}{v^{4}}+4 c\right),
\end{aligned}
$$

то каноническое преобразование между системами $1: 6: 1$ и $1: 6: 8$ приобретает вид [5]

$$
\begin{aligned}
u & =-\frac{P_{U}}{U}+\frac{P_{V}}{V}+\frac{\kappa_{1}}{U^{2}}+\frac{\kappa_{2}}{V^{2}}, \\
p_{u} & =-\frac{1}{4}\left(V^{2}-U^{2}\right)+\frac{P_{U}^{2}}{U^{2}}-\frac{P_{V}^{2}}{V^{2}}-2 \kappa_{1} \frac{P_{U}}{U^{3}}-2 \kappa_{2} \frac{P_{V}}{V^{3}}+\frac{\kappa_{1}^{2}}{U^{4}}-\frac{\kappa_{2}^{2}}{V^{4}}, \\
v & =2 \sqrt{\Omega}, \quad p_{v}=\sqrt{\Omega}\left(-\frac{P_{U}}{U}-\frac{P_{V}}{V}+\frac{\kappa_{1}}{U^{2}}-\frac{\kappa_{2}}{V^{2}}\right)-\frac{\kappa_{2}-\kappa_{1}}{2 \sqrt{\Omega}}, \\
U & =\frac{1}{2} \sqrt{\Gamma_{-}}, \quad P_{U}=-\frac{1}{2} \sqrt{\Gamma_{-}}\left(\frac{u}{2}+\frac{p_{v}}{v}-\frac{\kappa_{2}-\kappa_{1}}{v^{2}}\right)+2 \kappa_{1} \sqrt{\Gamma_{-}}, \\
V & =\frac{1}{2} \sqrt{\Gamma_{+}}, \quad P_{V}=\frac{1}{2} \sqrt{\Gamma_{+}}\left(\frac{u}{2}-\frac{p_{v}}{v}+\frac{\kappa_{2}-\kappa_{1}}{v^{2}}\right)-2 \kappa_{2} \sqrt{\Gamma_{+}} .
\end{aligned}
$$




\section{4. ОБЩЕЕ РЕШЕНИЕ ГАМИЛЬТОНИАНА \\ СИСТЕМЫ $1: 6: 8$ ПРИ $\gamma=0$}

При $\alpha=\beta=0$ можно построить преобразование (72), (73) в терминах переменных

$$
\begin{aligned}
Q_{1} & =\frac{1}{2}(U+V)^{2}, & P_{1} & =\frac{1}{2} \frac{P_{U}+P_{V}}{U+V}, \\
Q_{2} & =\frac{1}{2}(U-V)^{2}, & P_{2} & =\frac{1}{2} \frac{P_{U}-P_{V}}{U-V},
\end{aligned}
$$

которые задают разделение переменных для гамильтониана (48) системы $1: 6: 1$ в полиномиальном случае. Это дает замену переменных

$$
\begin{aligned}
& q_{1}=4 \frac{k_{2,0}+p_{v}^{2}}{v^{2}}-\frac{v^{2}}{2}-u^{2}-4 c \\
& p_{1}=\frac{-8 v p_{v} p_{u}+8 u p_{v}^{2}+2 v^{2} u^{3}+u\left(v^{4}+8 k_{2,0}\right)+8 c u v^{2}}{16 v\left(v p_{u}-2 u p_{v}\right)} \\
& q_{2}=4 \frac{-k_{2,0}+p_{v}^{2}}{v^{2}}-\frac{v^{2}}{2}-u^{2}-4 c \\
& p_{2}=\frac{-8 v p_{v} p_{u}+8 u p_{v}^{2}+2 v^{2} u^{3}+u\left(v^{4}-8 k_{2,0}\right)+8 c u v^{2}}{16 v\left(v p_{u}-2 u p_{v}\right)}
\end{aligned}
$$

которую можно применить к гамильтониану системы (53) при $\kappa_{1} \kappa_{2} \neq 0$, т.е. когда $k_{2,0}$ не является постоянной движения. Это преобразование, будучи ограниченным на $\kappa_{2}=$ $-\kappa_{1} \quad\left(\delta=-4 \kappa_{1}^{2}, \quad \gamma=0\right)$ задает координаты, которые разделяют переменные [11], [12] для уравнения Гамильтона-Якоби. В самом деле, получим

$$
H \equiv k_{1}=\frac{1}{16}\left(32 q_{1} p_{1}^{2}-q_{1}^{2}+32 q_{2} p_{2}^{2}-q_{2}^{2}-8 c\left(q_{1}+q_{2}\right)\right)-\kappa_{1}^{2} \frac{\left(q_{1}-q_{2}\right)}{4 k_{2,0}}
$$

где

$$
\begin{aligned}
k_{2,0} & =\frac{1}{8}\left(32 q_{1} p_{1}^{2}-q_{1}^{2}-32 q_{2} p_{2}^{2}+q_{2}^{2}+8 c\left(q_{2}-q_{1}\right)\right) \\
q_{1}^{\prime} & =4 q_{1} p_{1}+2 \kappa_{1}^{2} \frac{\left(q_{1}-q_{2}\right) q_{1} p_{1}}{k_{2,0}^{2}} \\
q_{2}^{\prime} & =4 q_{2} p_{2}-2 \kappa_{1}^{2} \frac{\left(q_{1}-q_{2}\right) q_{2} p_{2}}{k_{2,0}^{2}} .
\end{aligned}
$$

Вводя определения

$$
f\left(q_{i}, p_{i}\right) \equiv 32 q_{i} p_{i}^{2}-q_{i}^{2}-8 c q_{i}, \quad i=1,2,
$$

можно получить уравнение Гамильтона-Якоби в разделенных переменных

$$
f\left(q_{1}, p_{1}\right)^{2}-f\left(q_{2}, p_{2}\right)^{2}-32 \kappa_{1}^{2}\left(q_{1}-q_{2}\right)=16 k_{1}\left(f\left(q_{1}, p_{1}\right)-f\left(q_{2}, p_{2}\right)\right), \quad p_{i}=\frac{\partial S}{\partial q_{i}}
$$


По аналогии со случаем КК можно записать второй инвариант двумя эквивалентными способами,

$$
k_{2}^{2}=-2 \kappa_{1}^{2} q_{1}+\left(k_{2,0}+\frac{\kappa_{1}^{2}\left(q_{1}-q_{2}\right)}{2 k_{2,0}}\right)^{2}
$$

или

$$
k_{2}^{2}=-2 \kappa_{1}^{2} q_{2}+\left(k_{2,0}-\frac{\kappa_{1}^{2}\left(q_{1}-q_{2}\right)}{2 k_{2,0}}\right)^{2}
$$

с помощью которых можно исключить $\kappa_{1}^{2}\left(q_{1}-q_{2}\right) / k_{2,0}$ из равенств $(79),(84)$ и $(79),(85)$, соответственно. Далее, исключим $p_{1}$ и $p_{2}$ из получившихся выражений и равенств $(81)$, (82). В итоге будем иметь

$$
\begin{aligned}
q_{1}^{\prime}= & q_{1} \sqrt{\frac{q_{1}}{2}+4 c+4 \frac{k_{1}}{q_{1}}+\frac{2}{q_{1}} \sqrt{k_{2}^{2}+2 \kappa_{1}^{2} q_{1}} \times} \\
& \times\left(1+\frac{2 \kappa_{1}^{2}\left(q_{1}-q_{2}\right)}{\left(\sqrt{k_{2}^{2}+2 \kappa_{1}^{2} q_{1}}+\sqrt{k_{2}^{2}+2 \kappa_{1}^{2} q_{2}}\right)^{2}}\right), \\
q_{2}^{\prime}= & q_{2} \sqrt{\frac{q_{2}}{2}+4 c+4 \frac{k_{1}}{q_{2}}-\frac{2}{q_{2}} \sqrt{k_{2}^{2}+2 \kappa_{1}^{2} q_{2}} \times} \\
& \times\left(1-\frac{2 \kappa_{1}^{2}\left(q_{1}-q_{2}\right)}{\left(\sqrt{k_{2}^{2}+2 \kappa_{1}^{2} q_{1}}+\sqrt{k_{2}^{2}+2 \kappa_{1}^{2} q_{2}}\right)^{2}}\right) .
\end{aligned}
$$

Обшее решение уравнений $(86),(87)$ при $\kappa_{1}=0$ может быть выражено через эллиптические функции Вейерштрасса:

$$
\begin{aligned}
& q_{1}+\frac{8}{3} c=8 \wp\left(\xi-\xi_{1}, \frac{4}{3} c^{2}-\frac{k_{1}}{2}-\frac{k_{2}}{4}, \frac{c}{12}\left(2 k_{1}+k_{2}-\frac{32}{9} c^{2}\right)\right) \equiv 8 \wp_{1}, \\
& q_{2}+\frac{8}{3} c=8 \wp\left(\xi-\xi_{2}, \frac{4}{3} c^{2}-\frac{k_{1}}{2}+\frac{k_{2}}{4}, \frac{c}{12}\left(2 k_{1}-k_{2}-\frac{32}{9} c^{2}\right)\right) \equiv 8 \wp_{2} .
\end{aligned}
$$

При $\kappa_{1} \neq 0$, полагая в выражениях $(86),(87)$

$$
s_{1}=\sqrt{\frac{1}{2 \kappa_{1}^{2}} k_{2}^{2}+q_{1}}, \quad s_{2}=-\sqrt{\frac{1}{2 \kappa_{1}^{2}} k_{2}^{2}+q_{2}}
$$

и определяя переменную

$$
\widetilde{P}(s) \equiv \frac{1}{2}\left(s^{2}-\frac{k_{2}^{2}}{2 \kappa_{1}^{2}}\right)^{3}+4 c\left(s^{2}-\frac{k_{2}^{2}}{2 \kappa_{1}^{2}}\right)^{2}+\left(4 k_{1}+2 \sqrt{2} \kappa_{1} s\right)\left(s^{2}-\frac{k_{2}^{2}}{2 \kappa_{1}^{2}}\right),
$$

получаем уравнения

$$
s_{1}^{\prime}=\frac{\sqrt{\widetilde{P}\left(s_{1}\right)}}{s_{1}-s_{2}}, \quad s_{2}^{\prime}=-\frac{\sqrt{\widetilde{P}\left(s_{2}\right)}}{s_{1}-s_{2}}
$$


которые можно решить путем обрашения гиперэллиптических интегралов,

$$
\begin{aligned}
& \int_{\infty}^{s_{1}} \frac{d s}{\sqrt{\widetilde{P}(s)}}+\int_{\infty}^{s_{2}} \frac{d s}{\sqrt{\widetilde{P}(s)}}=k_{3}, \\
& \int_{\infty}^{s_{1}} \frac{s d s}{\sqrt{\widetilde{P}(s)}}+\int_{\infty}^{s_{2}} \frac{s d s}{\sqrt{\widetilde{P}(s)}}=\xi+k_{4} .
\end{aligned}
$$

Таким образом, если параметр $\delta$ произволен, а $\gamma=0$, то общее решение гамильтониана системы $1: 6: 8$ выражается через симметрические комбинации переменных $s_{1}, s_{2}$,

$$
u^{2}=-\frac{1}{2}\left(s_{1}^{2}+s_{2}^{2}\right)+\frac{k_{2}^{2}}{2 \kappa_{1}^{2}}+\left(\frac{s_{1}^{\prime}+s_{2}^{\prime}}{s_{1}+s_{2}}\right)^{2}-\frac{2 \sqrt{2} \kappa_{1}}{s_{1}+s_{2}}-4 c, \quad v^{2}=\frac{4 \sqrt{2} \kappa_{1}}{s_{1}+s_{2}}
$$

и представляет собой однозначную функцию комплексной переменной $\xi$. При $\kappa_{1}=0$ это решение вырождается и принимает вид

$$
u^{2}=-4\left(\wp_{1}+\wp_{2}\right)+\left(\frac{\wp_{1}^{\prime}-\wp_{2}^{\prime}}{\wp_{1}-\wp_{2}}\right)^{2}+\frac{k_{2,0}}{2\left(\wp_{2}-\wp_{1}\right)}-\frac{4}{3} c, \quad v^{2}=\frac{k_{2,0}}{\wp_{1}-\wp_{2}} .
$$

\section{5. ОБЩЕЕ РЕШЕНИЕ РАСШИРЕННОГО \\ ГАМИЛЬТОНИАНА СИСТЕМЫ $1: 6: 1$}

Для построения обшего решения гамильтониана системы $1: 6: 1$ можно опять использовать каноническое преобразование (72), (73).

При $\kappa_{1}=\kappa_{2}=0$ можно подставить выражения (96) в формулы (72), (73), получив при этом решение уравнений движения

$$
U^{2}+V^{2}=8\left(\wp_{1}+\wp_{2}-\frac{2}{3} c\right), \quad U V=4\left(\wp_{1}-\wp_{2}\right)
$$

Далее, при $\alpha \beta \neq 0$ и $\alpha=\beta$ получаются выражения

$$
\begin{aligned}
U^{2}+V^{2}= & -2 \sqrt{2}\left(s_{1}^{\prime}+s_{2}^{\prime}\right)+2\left(s_{1}^{2}+s_{2}^{2}+s_{1} s_{2}\right)-\frac{K_{2}^{2}}{\kappa_{1}^{2}} \\
U^{2} V^{2}= & -2 \sqrt{2}\left(s_{1}+s_{2}\right)\left(s_{1} s_{1}^{\prime}+s_{2} s_{2}^{\prime}-2 \kappa_{1}\right)+ \\
& +2\left(s_{1}+s_{2}\right)^{2}\left(s_{1}^{2}+s_{2}^{2}-\frac{3}{4} \frac{K_{2}^{2}}{\kappa_{1}^{2}}+4 c\right)
\end{aligned}
$$

которые зависят от симметрических комбинаций переменных $s_{1}, s_{2}$ и потому являются однозначными функциями $\xi$.

Так как гамильтониан (48) при $\alpha=\beta$ представляет собой четную функцию $\kappa_{1}$, можно применить каноническое преобразование (70), (71) при $\kappa_{1}=\kappa_{2}$ к выражениям (98), (99), получив при этом обшее решение гамильтониана системы $1: 6: 8$ в расширенном случае при $\delta=0$. 


\section{6. ЗАКЛЮЧЕНИЕ}

Ромейрас [22] описал процедуру, которая соотносит расширенный кубический потенциал $\widetilde{V}_{3}(u, v)=V_{3}(u, v)+\mu v^{-2} / 2$ в случае KK с расширенным потенциалом четвертого порядка $\widetilde{V}_{4}(u, v)=V_{4}(u, v)+\delta v^{-2} / 2$ в случае $1: 6: 8$. Это объясняет, почему комбинация $u^{2}+v^{2} / 2+4 c$ решений вида (95) идентична решению вида (43). Проблема распространения наших результатов на случай $\alpha \neq \beta$, т.е. на случай $\gamma \delta \neq 0$, остается открытой.

Благодарности. К.В., научный исследователь Фонда научных исследований Фландрии, благодарна организаторам за финансовую поддержку, которая позволила ей принять участие в конференции. Работа поддержана бельгийско-французской "Programme d'actions intégrées" (Tournesol T99/040), правительством Бельгии (IUAP P4/08), а также CEA.

\section{Список литературы}

[1] T. Bountis, H. Segur, F. Vivaldi. Phys. Rev. A. 1982. V. 25. P. 1257-1264.

[2] Y. F. Chang, M. Tabor, J. Weiss. J. Math. Phys. 1982. V. 23. P. 531-538.

[3] B. Grammaticos, B. Dorizzi, R. Padjen. Phys. Lett. A. 1982. V. 89. P. 111-113.

[4] A. P. Fordy. Physica D. 1991. V. 52. P. 204-210.

[5] S. Baker. Squared eigenfunction representation of integrable hierarchies. PhD Thesis. Leeds: University of Leeds, 1995.

[6] C. Verhoeven, M. Musette, R. Conte. J. Math. Phys. 2002. V. 43. P. 1906-1915; nlin.SI/0112030.

[7] B. Grammaticos, B. Dorizzi, A. Ramani. J. Math. Phys. 1983. V. 24. P. 2289-2295.

[8] J. Hietarinta. J. Math. Phys. 1984. V. 25. P. 1833-1844.

[9] J. Hietarinta. Phys. Rep. 1987. V. 147. P. 87-154.

[10] B. Grammaticos, B. Dorizzi, A. Ramani. J. Math. Phys. 1984. V. 25. P. 3470-3473.

[11] V. Ravoson, A. Ramani, B. Grammaticos. Phys. Lett. A. 1994. V. 191. P. 91-95.

[12] F. J. Romeiras. J. Math. Phys. 1995. V. 36. P. 3559-3565.

[13] С. В. Манаков. ЖЭТФ. 1973. Т. 65. С. 505-516.

[14] R. Garnier. Rendiconti del Circolo Matematico di Palermo. 1919. V. 43. P. 3559-3565.

[15] S. Wojciechowski. Physica Scripta. 1985. V. 31. P. 433-438.

[16] В. Г. Дринфельд, В. В. Соколов. Тр. семин. Л. С. Соболева. 1981. Т. 2. С. 5-9.

[17] S. Baker, V. Z. Enolskii, A. P. Fordy. Phys. Lett. A. 1995. V. 201. P. 167-174.

[18] J. Satsuma, R. Hirota. J. Phys. Soc. Japan. 1982. V. 51. P. 3390-3397.

[19] A. P. Fordy, J. Gibbons. J. Math. Phys. 1980. V. 21. P. 2508-2510.

[20] M. Btaszak, S. Rauch-Wojciechowski. J. Math. Phys. 1994. V. 35. P. 1693-1709.

[21] V. Ravoson, L. Gavrilov, R. Caboz. J. Math. Phys. 1993. V. 34. P. 2385-2393.

[22] F. J. Romeiras. J. Phys. A. 1995. V. 28. P. 5633-5642. 
Исправление к статье:

$\Phi$. Калоджеро. "Разрешимая задача трех тел и гипотезы Пенлеве"

(ТМФ. 2002. Т. 133. № 2. С. 149-159)

Таблица на с. 152 должна иметь следующий вид:

\begin{tabular}{|c|c|c|c|c|c|c|c|c|c|c|c|}
\hline$a_{12}$ & 0 & 0 & 0 & 0 & 0 & 0 & $-1 / 2$ & $-1 / 2$ & $-1 / 2$ & $-1 / 2$ & -1 \\
\hline$a_{23}$ & $-1 / 2$ & $-1 / 2$ & $-1 / 2$ & -1 & -1 & $-3 / 2$ & $-1 / 2$ & $-1 / 2$ & $-1 / 2$ & -1 & -1 \\
\hline$a_{31}$ & $-1 / 2$ & -1 & $-3 / 2$ & -1 & -2 & $-3 / 2$ & $-1 / 2$ & -1 & -2 & $-3 / 2$ & -1 \\
\hline$\beta_{12}$ & 0 & 0 & 0 & 0 & 0 & 0 & 1 & 1 & 1 & 1 & 2 \\
\hline$\beta_{23}$ & 1 & 1 & 1 & 2 & 2 & 3 & 1 & 1 & 1 & 2 & 2 \\
\hline$\beta_{31}$ & 1 & 2 & 3 & 2 & 4 & 3 & 1 & 2 & 4 & 3 & 2 \\
\hline$\gamma_{12}$ & 1 & 1 & 1 & 1 & 1 & 1 & 2 & 2 & 2 & 2 & $\infty$ \\
\hline$\gamma_{23}$ & 2 & 2 & 2 & $\infty$ & $\infty$ & -2 & 2 & 2 & 2 & $\infty$ & $\infty$ \\
\hline$\gamma_{31}$ & 2 & $\infty$ & -2 & $\infty$ & -1 & -2 & 2 & $\infty$ & -1 & -2 & $\infty$ \\
\hline$\Gamma$ & 3 & $\infty$ & -3 & -3 & -1 & -1 & $\infty$ & -3 & -1 & -1 & -1 \\
\hline
\end{tabular}

Редакция приносит свои извинения за эту ошибку. 